\title{
Time-like and space-like electromagnetic form factors of nucleons, a global description
}

\author{
Simone Pacetti* \\ Department of Physics, University of Perugia and I.N.F.N. Perugia, Italy \\ E-mail: simone.pacettidpq.infn.it

\section{Earle L. Lomon} \\ Center for Theoretical Physics and Laboratory for Nuclear Science and Department of Physics, \\ Massachusetts Institute of Technology, Cambridge, Massachusetts 02139
}

\begin{abstract}
The enlarged Lomon-Gari-Krümpelmann model of nucleon electromagnetic form factors, which embodies vector meson contributions and the asymptotic behavior of the perturbative QCD, has been extended to the time-like region by considering analytic Breit-Wigner formulae for broad resonances.

This analytic extension of the model has been used to perform global fit to all the nucleon electromagnetic form factor data, in the space-like and time-like region. The knowledge of the complex structure of the electromagnetic form factors enables a wide range of predictions on: asymptotic behaviors beyond the limits of the data, as well as on time-like observables connected with the real and imaginary parts.
\end{abstract}

XXI International Baldin Seminar on High Energy Physics Problems,

September 10-15, 2012

JINR, Dubna, Russia

\footnotetext{
${ }^{*}$ Speaker.
} 


\section{Introduction}

Nucleon electromagnetic form factors (EMFFs) describe modications of the pointlike photonnucleon vertex due to the structure of nucleons. The virtual photon, which mediates, in Born approximation, the electron-nucleon elastic scattering, interacting with single elementary charges, the quarks, is a powerful probe for the internal structure of the hadrons. Moreover, as the electromagnetic interaction is precisely calculable in QED, the dynamical content of each vertex can be extracted by comparing the data with the expectation, obtained under the pointlike hypothesis. The study of EMFFs is an essential step towards a deep understanding of the low-energy QCD dynamics. Nevertheless, even in case of nucleons, the available data are still incomplete.

\section{Nucleon electromagnetic form factors}

The elastic scattering of an electron by a nucleon, $e^{-} N \rightarrow e^{-} N$, is represented, in Born approximation, by the Feynman diagram of fig. $\square$ in the vertical direction. The 4-momentum, $q$, of the virtual photon is space-like, indeed, following the labelling of the figure and being $\theta$ the scattering angle, $q^{2}=-2 k_{1}^{0} k_{2}^{0}(1-\cos \theta) \leq 0$.

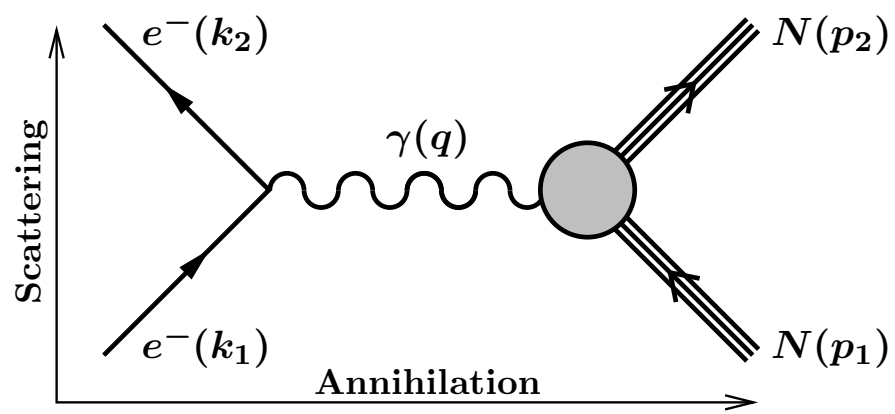

Figure 1: One-photon exchange Feynman diagram for scattering $e^{-} N \rightarrow e^{-} N$ and annihilation $e^{+} e^{-} \rightarrow N \bar{N}$.

By using the crossing symmetry, the annihilation $e^{+} e^{-} \rightarrow N \bar{N}$ is represented by the same Feynman

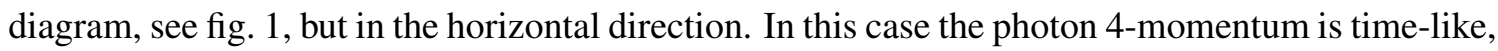
in fact: $q^{2}=4 k_{1}^{0} k_{2}^{0}=\left(2 k^{0}\right)^{2} \geq 0$, with $k_{1}^{0}=k_{2}^{0} \equiv k^{0}$.

The non-pointlike nucleon vertex, represented by the gray circle in fig. 四, is described by a nonconstant matrix $\Gamma^{\mu}$, which, by requiring Lorentz and gauge invariance, can be written in terms of two Lorentz scalar functions of the squared photon 4-momentum, $F_{1}^{N}\left(q^{2}\right)$ and $F_{2}^{N}\left(q^{2}\right)$, called Dirac and Pauli EMFFs, as [1]

$$
\Gamma^{\mu}=\gamma^{\mu} F_{1}^{N}\left(q^{2}\right)+\frac{i \sigma^{\mu v} q_{v}}{2 M_{N}} F_{2}^{N}\left(q^{2}\right),
$$

where $M_{N}$ is the nucleon mass $(N=n, p)$. These EMFFs are connected to the non-helicity-flip and the helicity-flip part of the hadronic current and are normalized, at $q^{2}=0$, to the total charge, $Q_{N}$ (in units of the positron charge), and the anomalous magnetic moment, $\kappa_{N}$ (in units of the Bohr magneton), of the nucleon

$$
F_{1}^{N}(0)=Q_{N}, \quad F_{2}^{N}(0)=\kappa_{N}
$$


The choice of a form factor pair to describe the two scalar degrees of freedom, allowed by Lorentz and gauge invariance, in the definition of the spin-1/2 electromagnetic current, is not unique. Besides the Dirac and Pauli EMFFs, we may use the so-called Sachs electric and magnetic form factors [2] (FFs)

$$
G_{E}^{N}\left(q^{2}\right)=F_{1}^{N}\left(q^{2}\right)+\frac{q^{2}}{4 M_{N}^{2}} F_{2}^{N}\left(q^{2}\right), \quad G_{M}^{N}\left(q^{2}\right)=F_{1}^{N}\left(q^{2}\right)+F_{2}^{N}\left(q^{2}\right)
$$

In the Breit frame (no energy exchanged, $q=(0, \vec{q})$ ) they correspond to the Fourier transformations of the charge and magnetic moment spatial distributions of the nucleon, the normalizations are

$$
G_{E}^{N}(0)=Q_{N}, \quad G_{M}^{N}(0)=\mu_{N}
$$

where $\mu_{N}=Q_{N}+\kappa_{N}$ is the nucleon magnetic moment (in units of the Bohr magneton).

As a consequence of microcausality, EMFFs are analytic functions of $q^{2}$, defined in the whole $q^{2}$ complex plane, with a cut, over the positive real axis, from the theoretical threshold $s_{\mathrm{th}}=\left(2 M_{\pi}\right)^{2}$, where $M_{\pi}$ is the pion mass, up to infinity, see fig. $\square$.

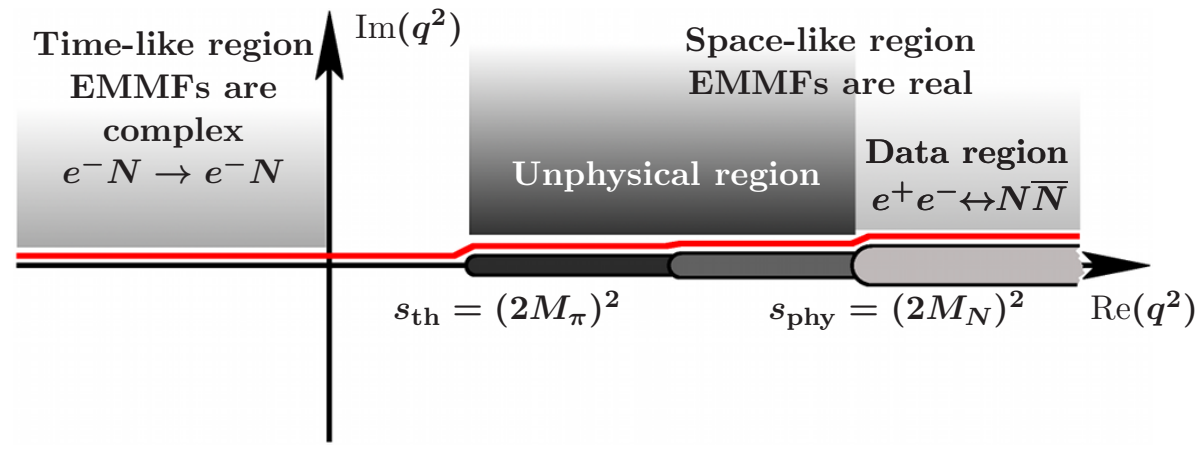

Figure 2: The $q^{2}$ complex plane. Cuts on the real axis and, experimentally accessible and forbidden regions are shown.

Following the optical theorem, the cut is the superposition of infinite discontinuities, three of them are sketched in fig. $\square$, corresponding to the opening of channels for every allowed intermediate state which couples with the virtual photon and the $N \bar{N}$ final state. The physical EMFFs are the limit over the upper edge of the real axis, red line in fig. [2, of such analytic functions and hence, below $s_{\text {th }}$, they are real, while, above that threshold, the EMFFs are complex.

The data in the two $q^{2}$ regions are extracted using observables from different processes. In reference to the sketch of fig. 凹, we have that:

- the space-like values of EMFFs are obtained from the elastic scattering differential cross section and polarization observables (see e.g. Ref. [3]);

- in the time-like region, above the so-called physical threshold $s_{\text {phy }}=\left(2 M_{N}\right)^{2}$, see fig. [■, the moduli of EMFFs are extracted by studying the angular distribution of the annihilation cross sections $\sigma\left(N \bar{N} \rightarrow e^{+} e^{-}\right)$and $\sigma\left(N \bar{N} \rightarrow e^{+} e^{-}\right)$; 
- the remaining part of the time-like region, namely that for: $0 \leq q^{2} \leq s_{\text {phy }}$, is called "unphysical region" because, even though the EMFFs are still well defined in such a region, they are not experimentally accessible.

The asymptotic behavior of the EMFFs in the space-like region is a $q^{2}$ power law that can be predicted in the framework of perturbative QCD (pQCD) either in terms of dimensional considerations [4], or in terms of minimal gluon exchange, among the constituent quarks, needed to share the photon transfer momentum [5].

More in detail, ignoring for the moment the logarithmic corrections of the strong coupling constant, as $q^{2} \rightarrow-\infty$, we have

$$
\left\{\begin{array} { l } 
{ F _ { 1 } ^ { N } ( q ^ { 2 } ) \sim ( - q ^ { 2 } ) ^ { - 2 } } \\
{ F _ { 2 } ^ { N } ( q ^ { 2 } ) \sim ( - q ^ { 2 } ) ^ { - 3 } }
\end{array} \Rightarrow \left\{\begin{array}{l}
G_{E}^{N}\left(q^{2}\right) \sim\left(-q^{2}\right)^{-2} \\
G_{M}^{N}\left(q^{2}\right) \sim\left(-q^{2}\right)^{-2}
\end{array}\right.\right.
$$

The Pauli EMFF $F_{2}^{N}\left(q^{2}\right)$, ruling the spin-flip part of the nucleon electromagnetic current, experiences a further suppression with respect to $F_{1}^{N}\left(q^{2}\right)$. The Sachs electric and magnetic form factors, instead, scale with same power law as $q^{2} \rightarrow-\infty$.

The asymptotic behavior in the time-like region, when $q^{2} \rightarrow \infty$, can be inferred by the space-like power law, using the Phragmèn-Lindelöf theorem [6]. Indeed, since the EMFFs are analytic and bounded functions of $q^{2}$ over the upper-half complex plane, they have the same asymptotic behavior over the arc of radius $\left|q^{2}\right|$ and subtended angle of $\pi$ radians, that sweeps the upper-half plane. Therefore the time-like asymptotic behavior, which is attained when $\left|q^{2}\right|$ diverges and $\arg \left(q^{2}\right)=0$, follows the same power law obtained from $\mathrm{pQCD}$ and counting rule in the space-like region, i.e. for $\arg \left(q^{2}\right)=\pi$. In particular, for the Sachs EMFFs, we have [7]

$$
\lim _{q^{2} \rightarrow-\infty} G_{E, M}^{N}\left(q^{2}\right)=\lim _{q^{2} \rightarrow+\infty} G_{E, M}^{N}\left(q^{2}\right) .
$$

\section{The model}

The model [8] that we use to parametrize the nucleon EMFFs is based on a primal idea due to Iachello, Jackson and Landé [9], then developed by Gari and Krümpelmann [10], and Lomon [11]. The two main ingredients of this model are: the vector-meson-dominance (VMD), for the low- $\left|q^{2}\right|$ regions (see, e.g., Ref. [12] for a review on VMD models), and the pQCD for the high- $\left|q^{2}\right|$ and asymptotic behavior. In the framework of VMD the coupling of the virtual photon with the nucleon vertex is mediated by vector mesons, hence the EMFFs are parametrized as a combination of vector meson propagators. As the momentum transfer increases, the propagator-like behavior is replaced by the power-law scaling predicted by pQCD. The transition between these different regimes is driven by the so-called hadron/quark FFs, acting at the vector meson-nucleon vertices. In order to have a simpler description in terms of vector-meson contributions, we consider the isospin components of the nucleon EMFFs. The Lomon model includes, besides the lightest vector mesons, excited states as well. So, the isovector (isospin $=1$ ) component has the $\rho$ and $\rho(1450)$, called simply $\rho^{\prime}$, contribution, while the isoscalar (isospin $=0$ ) component has the three contributions from: $\omega$ and $\omega(1420)$, called $\omega^{\prime}$, and $\phi$. 
In detail, for the isospin components of the Dirac and Pauli nucleon EMFFs, we have the following expressions

$$
\begin{aligned}
F_{1}^{\mathrm{iv}}\left(q^{2}\right)= & {\left[B W_{\rho}\left(q^{2}\right)+B W_{\rho^{\prime}}\left(q^{2}\right)\right] F_{1}^{\rho}\left(q^{2}\right)+\left[1-B W_{\rho}(0)-B W_{\rho^{\prime}}(0)\right] F_{1}^{D}\left(q^{2}\right) ; } \\
F_{2}^{\mathrm{iv}}\left(q^{2}\right)= & {\left[\kappa_{\rho} B W_{\rho}\left(q^{2}\right)+\kappa_{\rho^{\prime}} B W_{\rho^{\prime}}\left(q^{2}\right)\right] F_{2}^{\rho}\left(q^{2}\right)+\left[\kappa_{\mathrm{iv}}-\kappa_{\rho} B W_{\rho}(0)-\kappa_{\rho^{\prime}} B W_{\rho^{\prime}}(0)\right] F_{2}^{D}\left(q^{2}\right) ; } \\
F_{1}^{\mathrm{is}}\left(q^{2}\right)= & {\left[B W_{\omega}\left(q^{2}\right)+B W_{\omega^{\prime}}\left(q^{2}\right)\right] F_{1}^{\omega}\left(q^{2}\right)+B W_{\phi}\left(q^{2}\right) F_{1}^{\phi}\left(q^{2}\right) } \\
& +\left[1-B W_{\omega}(0)-B W_{\omega^{\prime}}(0)\right] F_{1}^{D}\left(q^{2}\right) ; \\
F_{2}^{\mathrm{is}}\left(q^{2}\right)= & {\left[\kappa_{\omega} B W_{\omega}\left(q^{2}\right)+\kappa_{\omega^{\prime}} B W_{\omega^{\prime}}\left(q^{2}\right)\right] F_{2}^{\omega}\left(q^{2}\right)+\kappa_{\phi} B W_{\phi}\left(q^{2}\right) F_{2}^{\phi}\left(q^{2}\right) } \\
& +\left[\kappa_{\mathrm{is}}-\kappa_{\omega} B W_{\omega}(0)-\kappa_{\omega^{\prime}} B W_{\omega^{\prime}}(0)\right] F_{2}^{D}\left(q^{2}\right),
\end{aligned}
$$

where:

- the vector-meson propagators are written as Breit-Wigner (BW) formulae, in pole approximation, in case of narrow resonances and with energy-dependent widths, in case of broad resonances

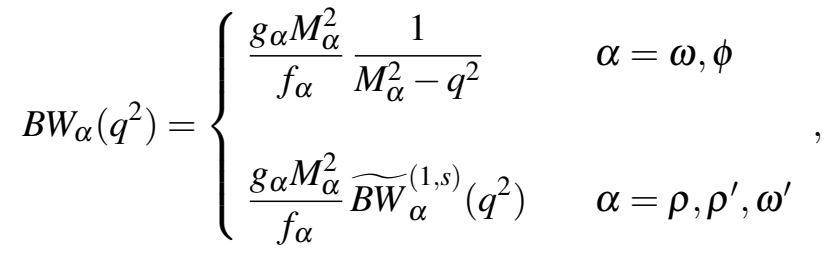

$M_{\alpha}$ is the mass and $M_{\alpha}^{2} g_{\alpha} / f_{\alpha}$ the coupling constant to the virtual photon and the nucleons of the vector meson $\alpha$. The three broad resonances $\rho, \rho^{\prime}$ and $\omega^{\prime}$ are described with an improved BW formula that has: energy-dependent width and the analytic properties required by microcausality and unitarity. We considered two possible descriptions, labelled with "1" or " $s "$, the case "1" is that with a minimal alteration from the non-relativistic BW form and the case " $s$ " from relativistic perturbation theory. We will discuss in more detail these analytic BW formulae in the next section;

- the functions $F_{1,2}^{\alpha}\left(q^{2}\right)$, are meson-nucleon FFs which describe the vertices $\alpha N N$, where the vector meson $\alpha$ couples with the nucleons. We have the same expression for all the vector mesons of $\rho$ and $\omega$ family, which is:

$$
F_{i}^{\alpha}\left(q^{2}\right)=f_{i}\left(q^{2}\right) \equiv \frac{\Lambda_{1}^{2}}{\Lambda_{1}^{2}-\tilde{q}^{2}}\left(\frac{\Lambda_{2}^{2}}{\Lambda_{2}^{2}-\tilde{q}^{2}}\right)^{i}, \quad \begin{aligned}
& i=1,2, \\
& \alpha=\rho, \rho^{\prime}, \omega, \omega^{\prime},
\end{aligned}
$$

while, in the case of the $\phi$ meson we consider an extra factor to force the Zweig suppression at low $q^{2}$ for the Dirac EMFF, so we have

$$
F_{1}^{\phi}\left(q^{2}\right)=f_{1}\left(q^{2}\right)\left(\frac{\tilde{q}^{2}}{\tilde{q}^{2}-\Lambda_{1}^{2}}\right)^{3 / 2}, \quad F_{2}^{\phi}\left(q^{2}\right)=f_{2}\left(q^{2}\right)\left(\frac{\Lambda_{1}^{2}}{\mu_{\phi}^{2}} \frac{\tilde{q}^{2}-\mu_{\phi}^{2}}{\tilde{q}^{2}-\Lambda_{1}^{2}}\right)^{3 / 2} .
$$

The free parameters $\Lambda_{1}$ and $\Lambda_{2}$ represent cut-offs for the general high-energy behavior and the helicity-flip respectively. Finally, $\tilde{q}^{2}$ is the transfer momentum squared which includes the QCD logarithmic corrections and it is defined as

$$
\tilde{q}^{2}=q^{2} \frac{\ln \left[\left(\Lambda_{D}^{2}-q^{2}\right) / \Lambda_{\mathrm{QCD}}^{2}\right]}{\ln \left(\Lambda_{D}^{2} / \Lambda_{\mathrm{QCD}}^{2}\right)}
$$


where $\Lambda_{\mathrm{QCD}}$ is the QCD scale and $\Lambda_{D}$ is a free parameter representing a cut-off which controls the asymptotic behavior of the quark-nucleon vertex;

- the non-resonant part of the amplitude, which accounts for the direct coupling of the virtual photon to the valence quarks of the nucleons and hence it is responsible for the asymptotic behavior, is described by the functions

$$
F_{i}^{D}\left(q^{2}\right)=\frac{\Lambda_{D}^{2}}{\Lambda_{D}^{2}-\tilde{q}^{2}}\left(\frac{\Lambda_{2}^{2}}{\Lambda_{2}^{2}-\tilde{q}^{2}}\right)^{i}, \quad i=1,2,
$$

$\Lambda_{D}$ and $\Lambda_{2}$, are the same parameters appearing in the meson nucleon FFs definition, and represent the scales of the asymptotia and of the helicity conservation, respectively, and $\tilde{q}^{2}$ is the QCD-corrected momentum transfer squared, defined in eq. (B.4);

- finally, $\kappa_{\alpha}$ is the ratio of tensor to vector coupling at $q^{2}=0$ in the $\alpha N N$ matrix element, while

$$
\kappa_{\mathrm{s}}=\kappa_{p}+\kappa_{n}, \quad \kappa_{\mathrm{v}}=\kappa_{p}-\kappa_{n} .
$$

are the isospin anomalous magnetic moments. The space-like asymptotic behavior $\left(q^{2} \rightarrow\right.$ $-\infty$ ) for the Dirac and Pauli EMFFs is driven by the $F_{1,2}^{D}\left(q^{2}\right)$ contribution, given in eq. (B.5)). In particular we get

$$
F_{1}^{\mathrm{iv}, \text { is }}\left(q^{2}\right) \underset{q^{2} \rightarrow-\infty}{\sim} \frac{1}{\left[q^{2} \ln \left(-q^{2} / \Lambda_{\mathrm{QCD}}^{2}\right)\right]^{2}}, \quad F_{2}^{\mathrm{iv}, \text { is }}\left(q^{2}\right) \underset{q^{2} \rightarrow-\infty}{\sim} \frac{F_{1}^{\mathrm{iv}, \text { is }}\left(q^{2}\right)}{-q^{2} \ln \left(-q^{2} / \Lambda_{\mathrm{QCD}}^{2}\right)},
$$

as required by the $\mathrm{pQCD}[4,5]$.

This analytic version of the Lomon-Gari-Krümpelmann (LGK) model can be naturally extended to the time-like region, for positive $q^{2}$, and so it is able to describe not only scattering data but also data on cross sections for the annihilation processes: $e^{+} e^{-} \leftrightarrow N \bar{N}$.

In fact, the BW formulae with energy-dependent widths are analytic in the whole $q^{2}$ complex plane with the cut $\left(s_{\mathrm{th}}=\left(2 M_{\pi}\right)^{2}, \infty\right)$, and the hadronic FFs of eqs. (B.2) and (B.J)), depending on $\tilde{q}^{2}$, for reasonable values of the cut-offs, have cuts but no poles on the physical Riemann sheet. Moreover, since the effect of the small widths of the narrow resonances $\omega$ and $\phi$ is negligible, we use for their propagators the non-relativistic expression.

\section{Analyticity of Breit-Wigner formulae}

The standard relativistic BW formula for an unstable particle of mass $M$ and constant width $\Gamma$ is the simple pole in $s \equiv q^{2}=M^{2}-i \Gamma M$, i.e.:

$$
B W(s)=\frac{1}{M^{2}-s-i \Gamma M} .
$$

If we consider an energy dependent width, immediately we face problems concerning the analyticity. In the case of $\rho$ meson, for instance, a realistic way to define an expression for the energy 
dependent width is to write down its decay rate to $\pi^{+} \pi^{-}$, assumed to be the dominant decay channel, as a function the $\rho$ mass squared, $s=M_{\rho}^{2}$,

$$
\Gamma_{s}^{\rho}(s)=\Gamma_{0}^{\rho} \frac{M_{\rho}^{2}}{s}\left(\frac{s-s_{\mathrm{th}}}{M_{\rho}^{2}-s_{\mathrm{th}}}\right)^{\frac{3}{2}}
$$

where: the subscript " $s$ " indicates the factor $1 / s$ appearing in the width definition, $\Gamma_{0}^{\rho}$ is the total width of the $\rho, M_{\rho}$ and $M_{\pi}$ are the $\rho$ and pion mass respectively, and $s_{\mathrm{th}}=\left(2 M_{\pi}\right)^{2}$.

It follows that the BW formula becomes

$$
B W_{s}(s)=\frac{s}{s\left(M_{\rho}^{2}-s\right)-i \Gamma_{0}^{\rho} M_{\rho}^{3}\left(\frac{s-s_{\mathrm{th}}}{M_{\rho}^{2}-s_{\mathrm{th}}}\right)^{\frac{3}{2}}} .
$$

In this form the BW has the "required" [13] discontinuity cut $\left(s_{\mathrm{th}}, \infty\right)$ and maintains a complex pole close to the non-relativistic one. Moreover, these are not the only complications introduced by using $\Gamma^{\rho}(s)$ instead of $\Gamma_{0}^{\rho}$, the power $3 / 2$ in the denominator and the factor $1 / s$, see eq. (4.2), generate also additional poles in the physical Riemann sheet which, to preserve analyticity, must be subtracted.

\subsection{Regularization of Breit-Wigner formulae}

We consider the general case of a BW formula which has $N$ poles, $\left\{p_{j}\right\}$, lying in the physical Riemann sheet. We define a regularization procedure which consists in subtracting these poles. In other words we add counterparts that at $s=p_{j}$ behave as the opposite of the $j^{\text {th }}$ pole. In more detail, we may define a regularized BW as

$$
\widetilde{B W}(s)=B W(s)-\sum_{j=1}^{N} \frac{R_{j}}{s-z_{j}}
$$

where $R_{j}$ is the residue of the $\mathrm{BW}$ with respect to the $j^{\text {th }}$ pole.

The same result can achieved by using the dispersion relations (DR) for the imaginary part. If the function $B W(s)$ is analytic in the $s$-complex plane, except for a discontinuity cut $\left(s_{\mathrm{th}}, \infty\right)$ and a finite number of isolated poles $\left\{p_{j}\right\}_{j=1}^{N}$, then the BW can written through the integral representation

$$
B W(s)=\frac{1}{\pi} \int_{s_{\mathrm{th}}}^{\infty} \frac{\operatorname{Im}\left[B W\left(s^{\prime}\right)\right]}{s^{\prime}-s} d s^{\prime}+2 i \pi \sum_{j=1}^{N} \frac{\operatorname{Res}\left[B W\left(s^{\prime}\right), p_{j}\right]}{s-p_{j}}
$$

It follows that the integral, in the left-hand side, coincides with the regularized BW defined in eq. (4.3)). Using the DR procedure we may regularize a BW formula without knowing its poles. We only need the imaginary part of the function $B W(s)$ that can be computed directly from the non-regularized expression. Once the imaginary part is known, the regularized expression is

$$
\widetilde{B W}(s)=\frac{1}{\pi} \int_{s_{\mathrm{th}}}^{\infty} \frac{\operatorname{Im}\left[B W\left(s^{\prime}\right)\right]}{s^{\prime}-s} d s^{\prime}
$$




\subsection{Two cases for $\Gamma(s)$}

We consider explicitly two expressions for $\Gamma(s)$, assuming only one main decay channel. Besides the form we discussed in Sec. 田, eq. (4.]), identified by the subscript "s", which was

$$
\Gamma_{s}(s)=\Gamma_{0} \frac{M^{2}}{s}\left(\frac{s-\tilde{s}_{\mathrm{th}}}{M^{2}-\tilde{s}_{\mathrm{th}}}\right)^{\frac{3}{2}},
$$

we consider also a simpler expression, closer to the non-relativistic form,

$$
\Gamma_{1}(s)=\Gamma_{0}\left(\frac{s-\tilde{s}_{\mathrm{th}}}{M^{2}-\tilde{s}_{\mathrm{th}}}\right)^{\frac{3}{2}},
$$

where $\tilde{s}_{\text {th }}$ is the threshold in each specific case. The subscript "1" in the expression of eq. (4.4), indicates that there is no extra factor $1 / s$ in the definition of the energy-dependent width.

As already discussed, the analyticity domain of the BW formulae is modified by the energydependent widths and, in particular, unwanted poles are introduced. To recover the regularity required by the theory, such poles must be subtracted. More in detail, in both cases with energydependent width $\Gamma_{1}(s)$ or $\Gamma_{s}(s)$, the BW formula acquires one real pole. We call these poles: $s_{s}$ and $s_{1}$ respectively (both of them are real and less than $\tilde{s}_{\mathrm{th}}$ ). The corresponding residues, $R_{s, 1}$, are

$$
R_{S}=\frac{s_{S}}{M^{2}-2 s_{S}+\frac{3}{2} \Gamma_{0} M^{3} \frac{\sqrt{s_{\mathrm{th}}-s_{s}}}{\left(M^{2}-\tilde{s}_{\mathrm{th}}\right)^{3 / 2}}}, \quad R_{1}=\frac{1}{-1+\frac{3}{2} \Gamma_{0} M \frac{\sqrt{\tilde{s}_{\mathrm{h}}-s_{1}}}{\left(M^{2}-\tilde{s}_{\mathrm{th}}\right)^{3 / 2}}} .
$$

Following eq. (4.3), the regularized BW formulae read

$$
\widetilde{B W}_{s, 1}(s)=B W_{s, 1}(s)-\frac{R_{s, 1}}{s-s_{s, 1}} .
$$

In particular, below the threshold $\tilde{s}_{\mathrm{th}}$ the $\mathrm{BW}$ formulae are real and we have

$$
\begin{aligned}
& \widetilde{B W}_{s}\left(s<\tilde{s}_{\mathrm{th}}\right)=\frac{s}{s\left(M^{2}-s\right)-\Gamma_{0} M^{3}\left(\frac{\tilde{s}_{\mathrm{th}}-s}{M^{2}-\tilde{s}_{\mathrm{th}}}\right)^{3 / 2}}-\frac{R_{s}}{s-s_{s}}, \\
& \widetilde{B W}_{1}\left(s<\tilde{s}_{\mathrm{th}}\right)=\frac{1}{M^{2}-s-\Gamma_{0} M\left(\frac{\tilde{s}_{\mathrm{th}}-s}{M^{2}-\tilde{s}_{\mathrm{th}}}\right)^{3 / 2}}-\frac{R_{1}}{s-s_{1}} .
\end{aligned}
$$

Above $\tilde{s}_{\text {th }}$ the BW formulae become complex, real and imaginary parts are obtained as limit of $\widetilde{B W}_{s, 1}(s)$ over the upper edge of the cut $\left(\tilde{s}_{\mathrm{th}}, \infty\right)$, the subtraction of the real poles $s_{s, 1}$ affects only the real parts, so we have

$$
\begin{aligned}
& \widetilde{B W}_{s}\left(s>\tilde{s}_{\mathrm{th}}\right)=\frac{s^{2}\left(M^{2}-s\right)}{s^{2}\left(M^{2}-s\right)^{2}+\Gamma_{0}^{2} M^{6}\left(\frac{s-\tilde{s}_{\mathrm{h}}}{M^{2}-\tilde{s}_{\mathrm{th}}}\right)^{3}}-\frac{R_{s}}{s-s_{s}}+i \frac{\Gamma_{0} M\left(\frac{s-\tilde{s}_{\mathrm{th}}}{M^{2}-\tilde{s}_{\mathrm{th}}}\right)^{3 / 2}}{\left(M^{2}-s\right)^{2}+\Gamma_{0}^{2} M^{2}\left(\frac{s-\tilde{s}_{\mathrm{th}}}{M^{2}-\tilde{s}_{\mathrm{th}}}\right)^{3}} \\
& \widetilde{B W}_{1}\left(s>\tilde{s}_{\mathrm{th}}\right)=\frac{M^{2}-s}{\left(M^{2}-s\right)^{2}+\Gamma_{0}^{2} M^{2}\left(\frac{s-\tilde{s}_{\mathrm{th}}}{M^{2}-\tilde{s}_{\mathrm{th}}}\right)^{3}}-\frac{R_{1}}{s-s_{1}}+i \frac{\Gamma_{0} M\left(\frac{s-\tilde{s}_{\mathrm{th}}}{M^{2}-\tilde{s}_{\mathrm{th}}}\right)^{3 / 2}}{\left(M^{2}-s\right)^{2}+\Gamma_{0}^{2} M^{2}\left(\frac{s-\tilde{s}_{\mathrm{th}}}{M^{2}-\tilde{s}_{\mathrm{th}}}\right)^{3}} .
\end{aligned}
$$

The parameters of the subtracted poles for the three broader vector mesons: $\rho, \rho^{\prime}$ and $\omega^{\prime}[14]$, are reported in table $\mathbb{\text { W. }}$ 


\begin{tabular}{l||c|c|c|c|c} 
Resonance & $M(\mathrm{GeV})$ & $\Gamma_{0}(\mathrm{GeV})$ & $\tilde{s}_{\mathrm{th}}$ & $s_{s}\left(\mathrm{GeV}^{2}\right)$ & $s_{1}\left(\mathrm{GeV}^{2}\right)$ \\
\hline \hline$\rho(770)$ & 0.7755 & 0.1491 & $\left(2 M_{\pi}\right)^{2}$ & 0.005953 & -11.63 \\
\hline$\rho(1450)$ & 1.465 & 0.400 & $\left(2 M_{\pi}\right)^{2}$ & 0.003969 & -29.43 \\
\hline$\omega(1420)$ & 1.425 & 0.215 & $\left(M_{\pi}+M_{\rho}\right)^{2}$ & 0.06239 & -19.46
\end{tabular}

Table 1: Parameters for the BW formulae of resonances: $\rho, \rho^{\prime}$ and $\omega^{\prime}$.

\section{The analytic extension}

The original LGK model, constructed in the space-like region, can be analytically continued in the time-like region using the regularized BW formulae obtained in Sec. 田. We consider then a new set of expressions for $F_{1,2}^{\mathrm{iv}}\left(q^{2}\right)$ and $F_{1,2}^{\text {is }}\left(q^{2}\right)$, homologous to those of eq. (B.D) where now we use, for the broader vector mesons, regularized BW formulae. Such BWs have the expected analytic structure and reproduce in both space-like and time-like regions the finite-width effect of broad resonances. These are the new expressions for the isospin components of nucleon EMFFs

$$
\begin{aligned}
F_{1, \text { case }}^{\text {iv }}\left(q^{2}\right)= & {\left[\widetilde{B W}_{\text {case }}^{\rho}\left(q^{2}\right)+\widetilde{B W}_{\text {case }}^{\rho^{\prime}}\left(q^{2}\right)\right] F_{1}^{\rho}\left(q^{2}\right)+\left[1-\widetilde{B W}_{\text {case }}^{\rho}(0)-\widetilde{B W}_{\text {case }}^{\rho^{\prime}}(0)\right] F_{1}^{D}\left(q^{2}\right) } \\
F_{2, \text { case }}^{\text {iv }}\left(q^{2}\right)= & {\left[\kappa_{\rho} \widetilde{B W}_{\text {case }}^{\rho}\left(q^{2}\right)+\kappa_{\rho^{\prime}} \widetilde{B W}_{\text {case }}^{\rho^{\prime}}\left(q^{2}\right)\right] F_{2}^{\rho}\left(q^{2}\right) } \\
& +\left[\kappa_{\mathrm{v}}-\kappa_{\rho} \widetilde{B W}_{\text {case }}^{\rho}(0)-\kappa_{\rho^{\prime}} \widetilde{B W}_{\text {case }}^{\rho^{\prime}}(0)\right] F_{2}^{D}\left(q^{2}\right) \\
F_{1, \text { case }}^{\text {is }}\left(q^{2}\right)= & {\left[B W^{\omega}\left(q^{2}\right)+\widetilde{B W}_{\text {case }}^{\omega^{\prime}}\left(q^{2}\right)\right] F_{1}^{\omega}\left(q^{2}\right)+B W^{\phi}\left(q^{2}\right) F_{1}^{\phi}\left(q^{2}\right)+} \\
& {\left[1-B W^{\omega}(0)-\widetilde{B W}_{\text {case }}^{\omega^{\prime}}(0)\right] F_{1}^{D}\left(q^{2}\right) } \\
F_{2, \text { case }}^{\text {is }}\left(q^{2}\right)= & {\left[\kappa_{\omega} B W^{\omega}\left(q^{2}\right)+\kappa_{\omega^{\prime}} \widetilde{B W}_{\text {case }}^{\omega^{\prime}}\left(q^{2}\right)\right] F_{2}^{\omega}\left(q^{2}\right)+\kappa_{\phi} B W^{\phi}\left(q^{2}\right) F_{2}^{\phi}\left(q^{2}\right)+} \\
& {\left[\kappa_{\mathrm{s}}-\kappa_{\omega} B W^{\omega}(0)-\kappa_{\omega^{\prime}} \widetilde{B W}_{\text {case }}^{\omega^{\prime}}(0)-\kappa_{\phi} B W^{\phi}(0)\right] F_{2}^{D}\left(q^{2}\right) }
\end{aligned}
$$

where case $=s$ and case $=1$ correspond to the parametrizations of the energy dependent width described in Sec. 4.2. Following eqs. (4.6) and (4.7) for the definition of $\widetilde{B W}\left(q^{2}\right)$, and including the coupling constants, we have

$$
\widetilde{B W}_{\text {case }}^{\beta}\left(q^{2}\right)=\left\{\begin{array}{ll}
\frac{g_{\beta} M_{\beta}^{2}}{f_{\beta}}\left[\frac{q^{2}}{\left.q^{2}\left(M_{\beta}^{2}-q^{2}\right)-i \Gamma_{0}^{\beta} M_{\beta}^{3}\left(\frac{q^{2}-s_{\mathrm{th}}^{\beta}}{M_{\beta}^{2}-s_{\mathrm{th}}^{\beta}}\right)^{3 / 2}-\frac{R_{s}^{\beta}}{q^{2}-s_{s}^{\beta}}\right]}\right. & \text { case }=s \\
\frac{g_{\beta} M_{\beta}^{2}}{f_{\beta}}\left[\frac{1}{M_{\beta}^{2}-q^{2}-i \Gamma_{0}^{\beta} M_{\beta}\left(\frac{q^{2}-s_{\mathrm{th}}^{\beta}}{M_{\beta}^{2}-s_{\mathrm{th}}^{\beta}}\right)^{3 / 2}}-\frac{R_{1}^{\beta}}{q^{2}-s_{1}^{\beta}}\right] & \text { case }=1
\end{array},\right.
$$

with: $\beta=\rho, \rho^{\prime}, \omega^{\prime}$ (parameters in table $\mathbb{W}$ ) and the residues $R_{s, 1}^{\beta}$ are given in eq. (4.5)).

The regularized BW formulae have the same high-energy behavior of the non-relativistic ones. In 
fact, as $\left|q^{2}\right| \rightarrow \infty$, the function $\widetilde{B W}_{\text {case }}^{\beta}\left(q^{2}\right)$ vanishes like $1 / q^{2}$, more in detail we have

$$
\widetilde{B W}_{\text {case }}^{\beta}\left(q^{2}\right) \underset{\left|q^{2}\right| \rightarrow \infty}{\sim} \begin{cases}\frac{g_{\beta} M_{\beta}^{2}}{f_{\beta}} \frac{1-R_{s}^{\beta}}{q^{2}} & \text { case }=s \\ -\frac{g_{\beta} M_{\beta}^{2}}{f_{\beta}} \frac{R_{1}^{\beta}}{q^{2}} & \text { case }=1\end{cases}
$$

It is interesting to notice that in both cases the expected behavior is ensured by the subtracted pole, in particular, the asymptotic limit of $q^{2} \cdot \widetilde{B W}_{\text {case }}^{\beta}\left(q^{2}\right)$ is proportional to $\left(1-R_{s}^{\beta}\right)$ and $R_{1}^{\beta}$, respectively.

\section{Results}

We have considered nine sets of data, six of them lie in the space-like region [15] and three in the time-like region [16-24]. The data are on the Sachs EMFFs and their ratios. The global $\chi^{2}$ is the sum of nine contributions, $\chi_{i}^{2}$, one for each set

$$
\chi^{2}=\sum_{i=1}^{9} \tau_{i} \cdot \chi_{i}^{2}, \quad \text { with: } \chi_{i}^{2}=\sum_{k=1}^{N_{i}}\left(\frac{Q_{i}\left(q_{k}^{2}\right)-v_{k}^{i}}{\delta v_{k}^{i}}\right)^{2}
$$

where the coefficients $\tau_{i}$ weight the $i^{\text {th }}$ contribution, we use $\tau_{i}=1$ or $\tau_{i}=0$ to include or exclude the $i^{\text {th }}$ data set, $Q_{i}\left(q^{2}\right)$ stands for the physical observable, function of $q^{2}$, and the set $\left\{q_{k}^{2}, v_{k}^{i}, \delta v_{k}^{i} ; N_{i}\right\}$ represents the corresponding data; $v_{k}^{i}$ is the $k^{\text {th }}$ value $\left(k=1, \ldots, N_{i}\right)$ of the quantity $Q_{i}(i=1, \ldots, 9)$

\begin{tabular}{|c|c|c|c|c|c|c|}
\hline & \multirow{2}{*}{$Q_{i}$} & \multirow{2}{*}{$N_{i}$} & \multicolumn{4}{|c|}{$\operatorname{minimum} \chi_{i}^{2}$} \\
\hline & & & $\begin{array}{c}\text { case }=s \\
\text { With } B_{A} B_{A R}\end{array}$ & $\begin{array}{c}\text { case }=1 \\
\text { With } B A B A R\end{array}$ & $\begin{array}{c}\text { case }=s \\
\text { No } B_{A} B_{A R}\end{array}$ & $\begin{array}{c}\text { case }=1 \\
\text { No } B_{A} B_{A R}\end{array}$ \\
\hline \multirow{6}{*}{ 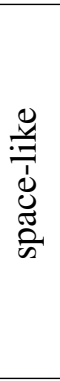 } & $G_{M}^{p}$ & 68 & 48.7 & 50.1 & 54.6 & 60.8 \\
\hline & $G_{E}^{p}$ & 36 & 30.4 & 27.6 & 26.2 & 35.0 \\
\hline & $G_{M}^{n}$ & 65 & 154.6 & 154.2 & 158.2 & 167.0 \\
\hline & $G_{E}^{n}$ & 14 & 22.7 & 23.2 & 24.1 & 26.0 \\
\hline & $\mu_{p} G_{E}^{p} / G_{M}^{p}$ & 25 & 13.9 & 12.9 & 10.6 & 14.4 \\
\hline & $\mu_{n} G_{E}^{n} / G_{M}^{n}$ & 13 & 11.3 & 10.7 & 8.2 & 8.9 \\
\hline \multirow{4}{*}{ 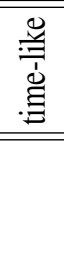 } & $\left|G_{\mathrm{eff}}^{p}\right|$ & $61(28)$ & 162.5 & 166.7 & 62.2 & 35.0 \\
\hline & $\left|G_{\text {eff }}^{n}\right|$ & 5 & 8.4 & 6.3 & 3.2 & 0.3 \\
\hline & Total & $285(252)$ & 452.5 & 451.7 & 347.3 & 347.4 \\
\hline & \multicolumn{2}{|c|}{ Normalized $\chi^{2}$} & 1.66 & 1.66 & 1.45 & 1.45 \\
\hline
\end{tabular}
measured at $q^{2}=q_{k}^{2}$, with error $\delta v_{k}^{i}$.

Table 2: Measured quantities, numbers of data points and $\chi^{2}$ contributions. The values in parentheses indicate the number of data points in the case "No BABAR", see the text.

We have considered four cases corresponding to the two different possible parametrizations for the 
$\mathrm{BW}$ formulae, the so-called case $=1$ and case $=s$, and two possible sets of time-like data, including and not including the $B_{A} B_{A R}$ data [24]. The possibility of excluding the $B_{A} B A R$ data has been taken into account because those data are the only obtained using the initial state radiation technique, that is, they have been extracted from the cross section of the radiative process $e^{+} e^{-} \rightarrow p \bar{p} \gamma$, where the photon is emitted by one of the initial lepton. The theoretical error in using this technique could be underestimated although relevant calculations have been made [25].

The complete list of observables is reported in table $\square$, the number of data points and the corresponding minimum $\chi^{2}$ are also shown.

Data and fits, black and gray curves correspond to case $=1$ and case $=s$ respectively, solid and dashed line to the cases with and without $B A B A R$ data respectively, are shown in figs. AW. In the space-like region the electric Sachs EMFFs are normalized to the dipole

$$
G_{D}\left(q^{2}\right)=\left[1-q^{2} /\left(0.71 \mathrm{GeV}^{2}\right)\right]^{-2},
$$

while magnetic EMFFs are also normalized to the magnetic moments. The dipole normalization decreases the range of variation and allows a quick estimate of the deviations from the dipole form itself. The quantity $R_{N}$ is the ratio defined as: $R_{N}=G_{E}^{N} / G_{M}^{N}$, for the nucleon $N$, that stands for both neutron and proton, hence there are six space-like observables.

The time-like effective FF, $\left|G_{\text {eff }}^{N}\right|$, is defined in terms of the total cross section, $\sigma\left(e^{+} e^{-} \rightarrow N \bar{N}\right)$, as

$$
\left|G_{\mathrm{eff}}^{N}\left(q^{2}\right)\right|=\left[\frac{\sigma\left(e^{+} e^{-} \rightarrow N \bar{N}\right)}{\frac{4 \pi \alpha^{2}}{3 q^{2}} \sqrt{1-\frac{4 M_{N}^{2}}{q^{2}}}\left(1+\frac{2 M_{N}^{2}}{q^{2}}\right)}\right]^{1 / 2},
$$

where the kinematic factor at denominator is the Born cross section for a pointlike nucleon. The same quantity can be expressed through the EMFFs, $G_{E}^{N}$ and $G_{M}^{N}$, i.e., considering the matrix element given in eq. (‥J) and the definitions of eq. (‥2), we have

$$
\left|G_{\mathrm{eff}}^{N}\left(q^{2}\right)\right|=\left(\left|G_{M}^{N}\left(q^{2}\right)\right|^{2}+\frac{2 M_{N}^{2}}{q^{2}}\left|G_{E}^{N}\left(q^{2}\right)\right|^{2}\right)^{1 / 2}\left(1+\frac{2 M_{N}^{2}}{q^{2}}\right)^{-1 / 2} .
$$

This is the relation that we use to fit the data on $\left|G_{\mathrm{eff}}^{N}\right|$ for both proton-antiproton and neutronantineutron production.

As a consequence of near-threshold flat cross sections, the effective nucleon FFs, extracted by the total cross section data through the relation of eq. (6. D), see data in figs. 8 and $\mathbb{0}$, have a steep enhancement when $q^{2} \rightarrow\left(2 M_{N}\right)^{2}$. Such a steep growth is in contrast with theory that predicts smooth behavior of nucleon EMFFs across the physical threshold, where, moreover, the formula of eq. (6. (ل) should be corrected to account for $N \bar{N}$ final state interaction. In particular, in case of $p \bar{p}$ we have to consider, not only the Coulomb correction, due to the electromagnetic attractive interaction [26], but also the effect of strong interaction [27] and the interplay between them, whose form is not well known. For that reason we included in the present analysis only time-like data with: $q^{2} \geq 4 \mathrm{GeV}^{2}$.

The free parameters of this model are:

- three cut-offs: $\Lambda_{1}, \Lambda_{2}$ and $\Lambda_{D}$ which parametrize the hadronic FFs and control the transition from non-perturbative to perturbative QCD regime; 
- five pairs of vector meson anomalous magnetic moments and photon couplings $\left(\kappa_{\alpha}, g_{\alpha} / f_{\alpha}\right)$, with $\alpha=\rho, \rho^{\prime}, \omega, \omega^{\prime}$, and $\phi$.

The values for these 13 free parameters together with the fixed parameters are reported in table B]. The fixed parameters describe dynamical quantities and well known properties of the intermediate vector mesons. For the QCD scale, we use the value $\Lambda_{\mathrm{QCD}}=0.15 \mathrm{GeV}$ in all cases but for the case $=1$, without $B A B A R$ data, where instead: $\Lambda_{\mathrm{QCD}}=0.10 \mathrm{GeV}$. Such a reduced value is motivated by the analyticity requirement of having no real poles in meson-nucleon and quark-nucleon FFs (Sec. [1). As $\Lambda_{\mathrm{QCD}}=0.15 \mathrm{GeV}$ is closer to the values preferred by high energy experiments, it suggests that case $=s$ is the more physical model. Another reason to prefer it on physical grounds is that the width formula of the vector meson decay is determined by relativistic perturbation theory. Case $=1$ was chosen because it is a simpler relativistic modification of the non-relativistic BW formula.

\begin{tabular}{|c|c|c|c|c|}
\hline Parameter & $\begin{array}{c}\text { case }=s \\
\text { With } B A B A R\end{array}$ & $\begin{array}{c}\text { case }=1 \\
\text { With } B A B A R\end{array}$ & $\begin{array}{c}\text { case }=s \\
\text { No } B_{A} B A R\end{array}$ & $\begin{array}{c}\text { case }=1 \\
\text { No } B A B A R\end{array}$ \\
\hline$g_{\rho} / f_{\rho}$ & 2.766 & 2.410 & 0.9029 & 0.4181 \\
\hline$\kappa_{\rho}$ & -1.194 & -1.084 & 0.8267 & 0.6885 \\
\hline$M_{\rho}(\mathrm{GeV})$ & \multicolumn{4}{|c|}{0.7755 (fixed) } \\
\hline$\Gamma_{\rho}(\mathrm{GeV})$ & \multicolumn{4}{|c|}{0.1491 (fixed) } \\
\hline$g_{\omega} / f_{\omega}$ & -1.057 & -1.043 & -0.2308 & -0.4894 \\
\hline$\kappa_{\omega}$ & -3.240 & -3.317 & -9.859 & -1.398 \\
\hline$M_{\omega}(\mathrm{GeV})$ & \multicolumn{4}{|c|}{0.78263 (fixed) } \\
\hline$g_{\phi} / f_{\phi}$ & 0.1871 & 0.1445 & 0.0131 & 0.1156 \\
\hline$\kappa_{\phi}$ & -2.004 & -3.045 & 37.218 & -0.2613 \\
\hline$M_{\phi}(\mathrm{GeV})$ & \multicolumn{4}{|c|}{1.019 (fixed) } \\
\hline$\mu_{\phi}(\mathrm{GeV})$ & \multicolumn{4}{|c|}{20.0 (fixed) } \\
\hline$g_{\omega^{\prime}} / f_{\omega^{\prime}}$ & 2.015 & 1.974 & 1.265 & 1.649 \\
\hline$\kappa_{\omega^{\prime}}$ & -2.053 & -2.010 & -2.044 & -0.6712 \\
\hline$M_{\omega^{\prime}}(\mathrm{GeV})$ & \multicolumn{4}{|c|}{1.425 (fixed) } \\
\hline$\Gamma_{\omega^{\prime}}(\mathrm{GeV})$ & \multicolumn{4}{|c|}{0.215 (fixed) } \\
\hline $\bar{~} \overline{g_{\rho^{\prime}} / f_{\rho^{\prime}}}$ & -3.475 & -3.274 & -0.8730 & -0.0369 \\
\hline$\kappa_{\rho^{\prime}}$ & -1.657 & -1.724 & -2.832 & -104.35 \\
\hline$M_{\rho^{\prime}}(\mathrm{GeV})$ & \multicolumn{4}{|c|}{1.465 (fixed) } \\
\hline$\Gamma_{\rho^{\prime}}(\mathrm{GeV})$ & \multicolumn{4}{|c|}{0.400 (fixed) } \\
\hline $\bar{~} \Lambda_{1}(\mathrm{GeV})$ & 0.4801 & 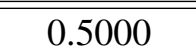 & 0.6474 & 0.6446 \\
\hline$\Lambda_{2}(\mathrm{GeV})$ & 3.0536 & 3.0562 & 3.0872 & 3.6719 \\
\hline$\Lambda_{D}(\mathrm{GeV})$ & 0.7263 & 0.7416 & 0.8573 & 0.8967 \\
\hline$\Lambda_{\mathrm{QCD}}(\mathrm{GeV})$ & \multicolumn{3}{|c|}{0.150} & 0.100 \\
\hline
\end{tabular}

Table 3: Best values of fit parameters and constants. 


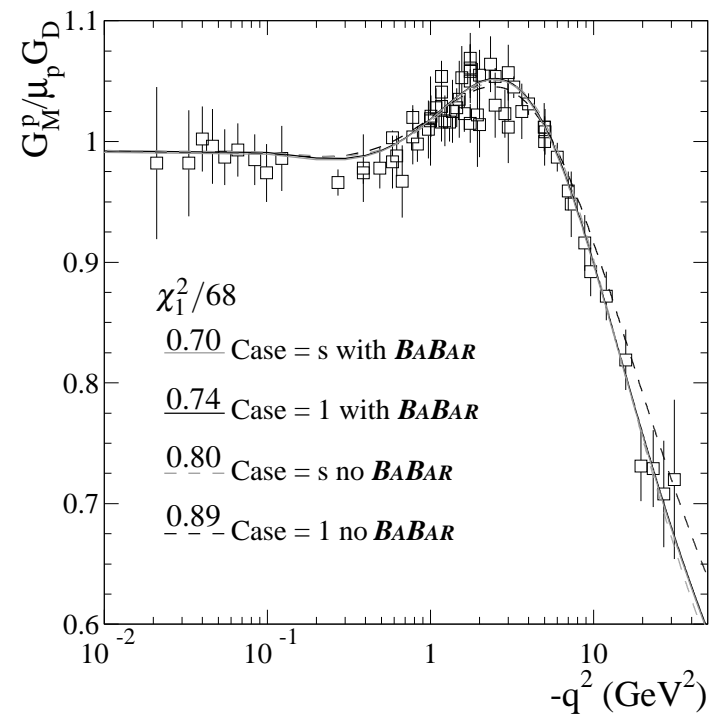

Figure 3: Space-like magnetic proton EMFF normalized to the dipole and $\mu_{p}$, in case $=1$ and case $=s$, including and not the $B A B A R$ data.

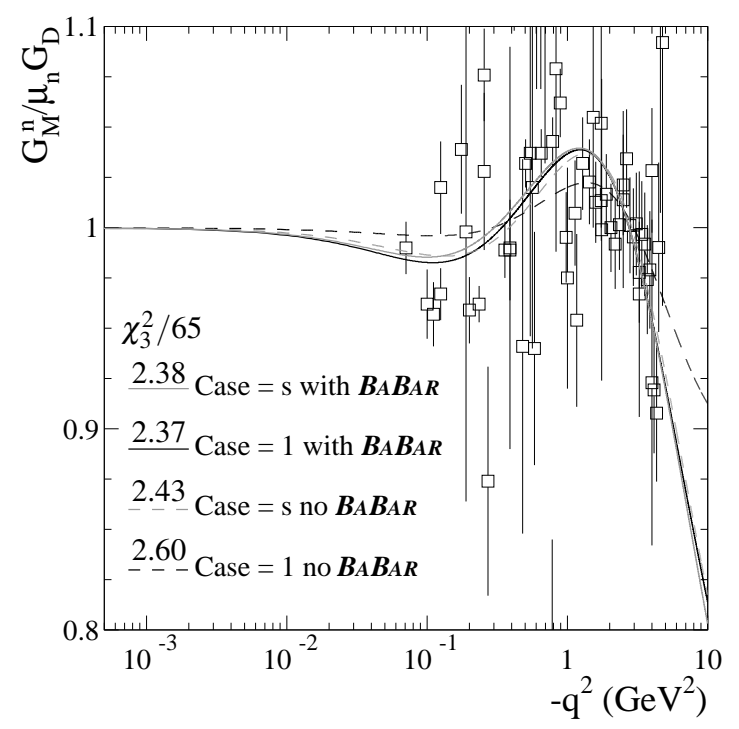

Figure 5: Space-like magnetic neutron EMFF normalized to the dipole and $\mu_{n}$, in case $=1$ and case $=s$, including and not the $B A B A R$ data.

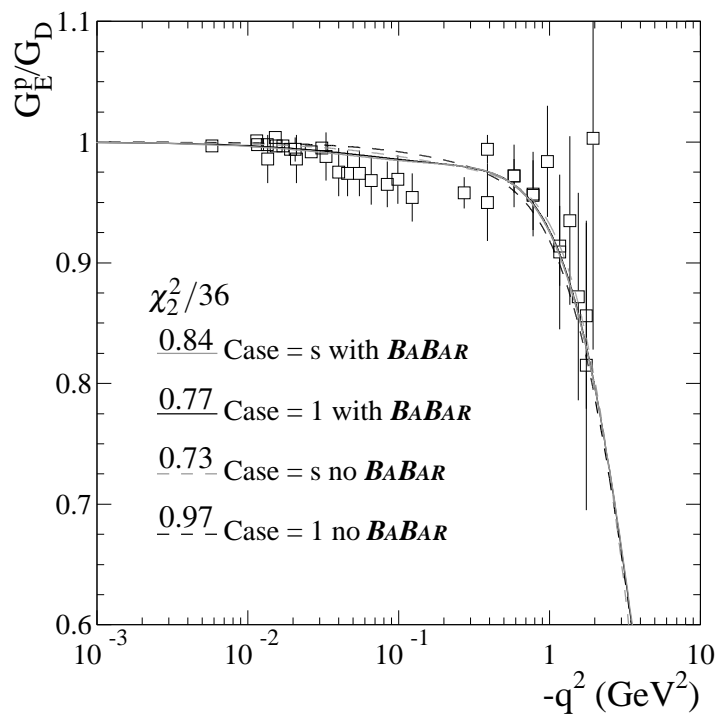

Figure 4: Space-like electric proton EMFF normalized to the dipole, in case $=1$ and case $=s$, including and not the $B A B A R$ data.

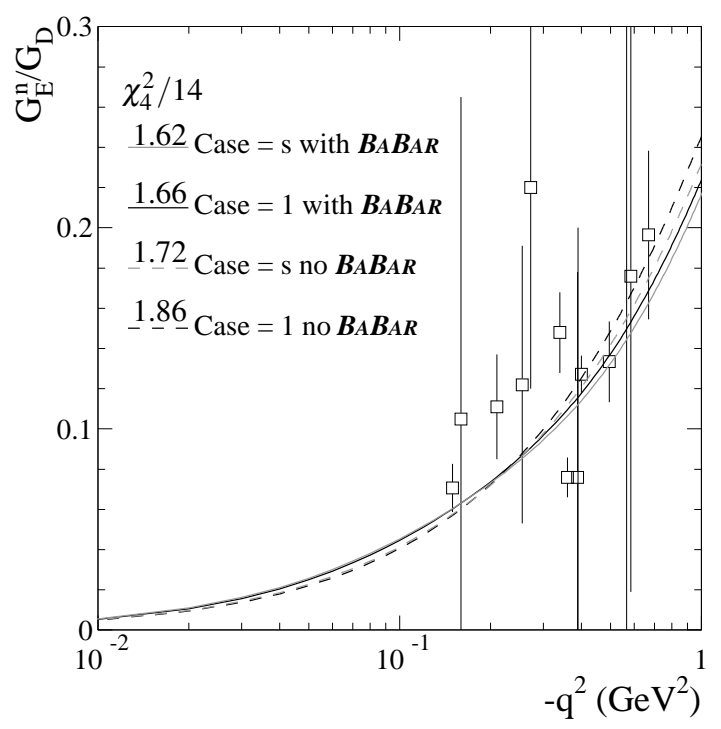

Figure 6: Space-like electric neutron EMFF normalized to the dipole, in case $=1$ and case $=s$, including and not the $B A B A R$ data.

The charge proton root mean square radii obtained in the four considered cases are

$$
\bar{r}_{p}=\sqrt{\left\langle r_{p}^{2}\right\rangle}=\left[\frac{1}{6} \frac{d G_{E}^{p}\left(q^{2}\right)}{d q^{2}}\right]_{q^{2}=0}^{1 / 2}= \begin{cases}0.876 \mathrm{fm} & \text { case }=s \text { with } B A B A R \\ 0.863 \mathrm{fm} & \text { case }=1 \text { with } B A B A R \\ 0.834 \mathrm{fm} & \text { case }=s \text { no } B A B A R \\ 0.819 \mathrm{fm} & \text { case }=1 \text { no } B A B A R\end{cases}
$$




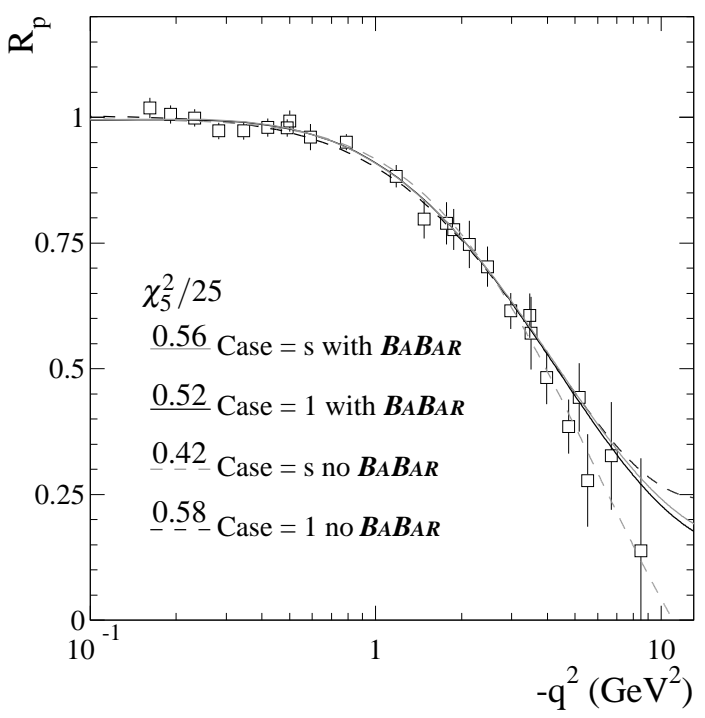

Figure 7: $\quad$ Space-like ratio $R_{p}=\mu_{p} G_{E}^{p} / G_{M}^{p}$ normalized to $\mu_{p}$, in case $=1$ and case $=s$, including and not the $B A B A R$ data.

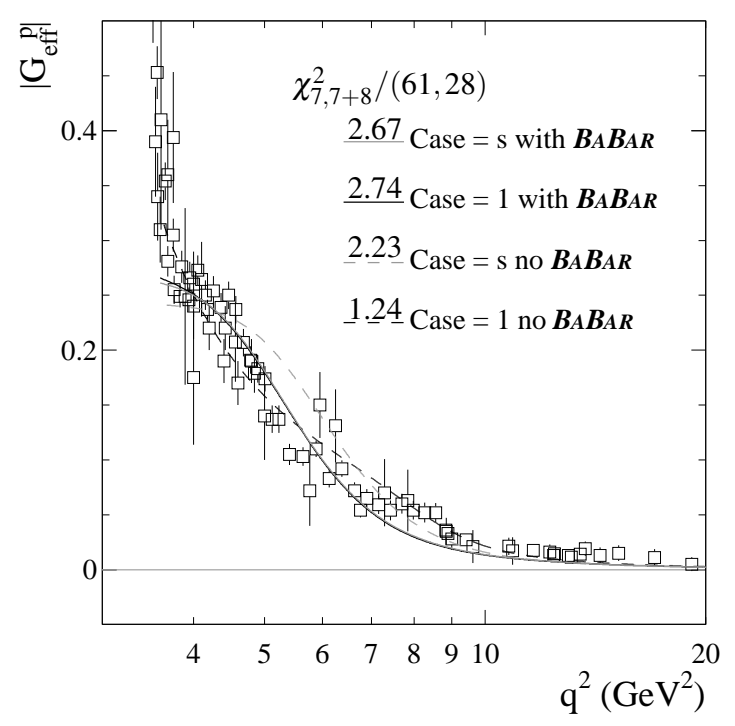

Figure 9: Time-like effective proton FF data (nine sets [16-24]) and fit, in case $=1$ and case $=s$, including and not the $B A B A R$ data.

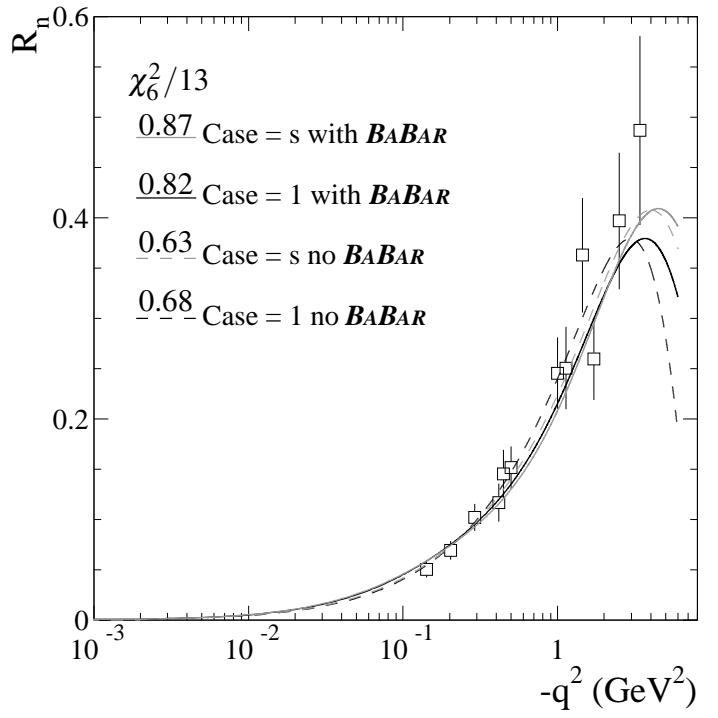

Figure 8: Space-like ratio $R_{n}=\mu_{n} G_{E}^{n} / G_{M}^{n}$ normalized to $\mu_{n}$, in case $=1$ and case $=s$, including and not the $B A B A R$ data.

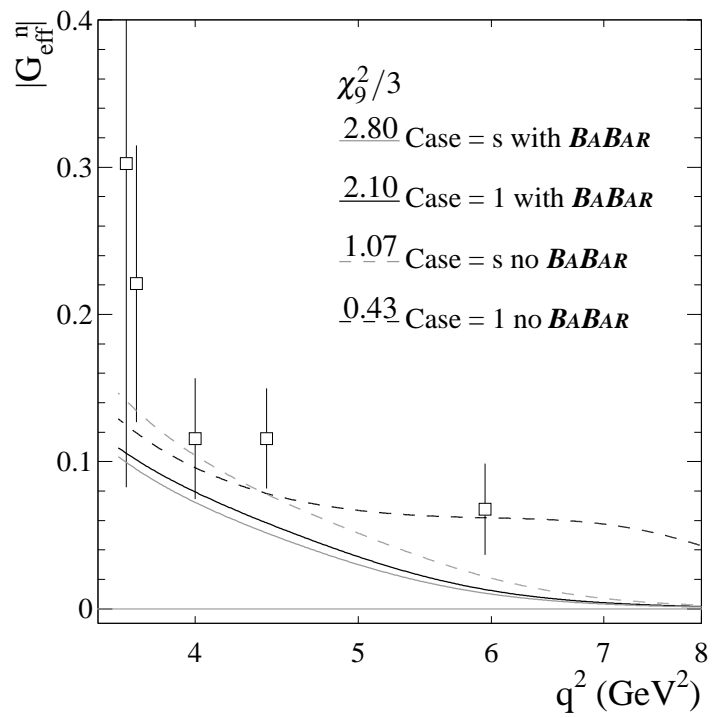

Figure 10: Time-like effective neutron FF data (only FENICE [16]) and fit, in case $=1$ and case $=s$, including and not the $B A B A R$ data.

to be compared with the Particle Data Group (PDG) value [14]

$$
\bar{r}_{p}^{\mathrm{PDG}}=0.877 \pm 0.005 \mathrm{fm} .
$$




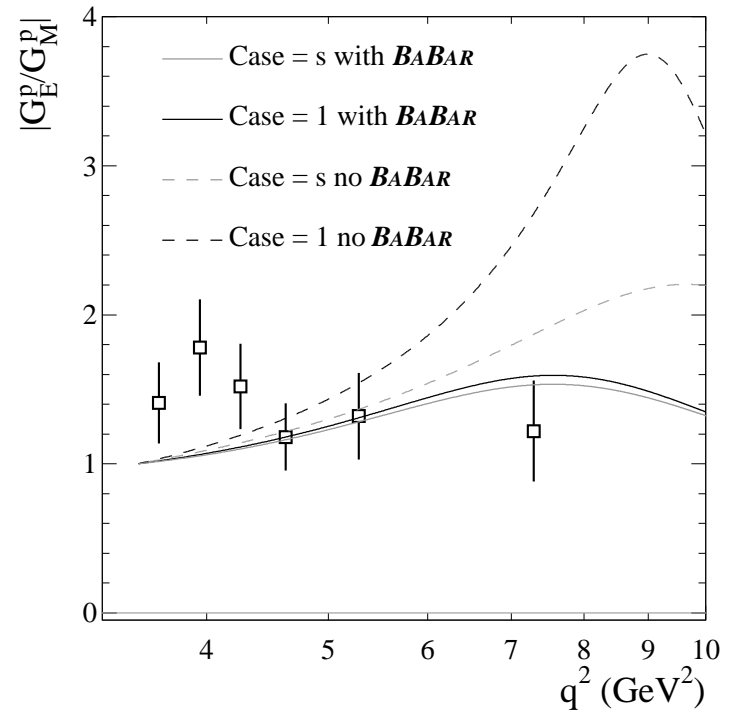

Figure 11: Modulus of the ratio $G_{E}^{p} / G_{M}^{p}$, data [24] and prediction, in the time-like region, in case $=1$ and case $=s$, including and not the $B A B A R$ data.

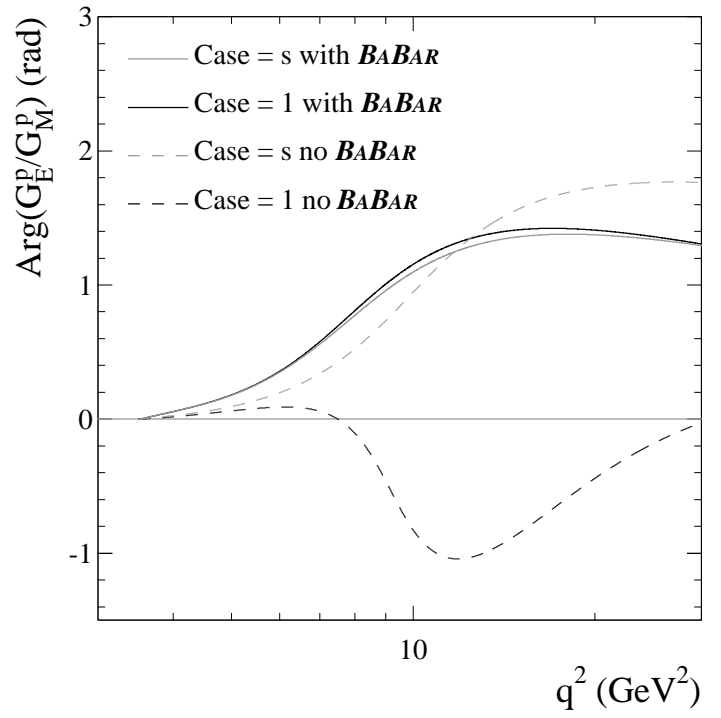

Figure 12: Prediction for the phase of the ratio $G_{E}^{p} / G_{M}^{p}$, in the time-like region, in case $=1$ and case $=s$, including and not the BABAR data.

In the case of the neutron, the mean square radii are

$$
\left\langle r_{n}^{2}\right\rangle=\left.\frac{1}{6} \frac{d G_{E}^{n}\left(q^{2}\right)}{d q^{2}}\right|_{q^{2}=0}= \begin{cases}-0.117 \mathrm{fm}^{2} & \text { case }=s \text { with } B_{A} B A R \\ -0.117 \mathrm{fm}^{2} & \text { case }=1 \text { with } B_{A} B A R \\ -0.112 \mathrm{fm}^{2} & \text { case }=s \text { no } B A B A R \\ -0.112 \mathrm{fm}^{2} & \text { case }=1 \text { no } B A B A R\end{cases}
$$

and the PDG value is [14]

$$
\left\langle r_{n}^{2}\right\rangle^{\mathrm{PDG}}=-0.1161 \pm 0.0022 \mathrm{fm}^{2} .
$$

\section{Discussion}

The LGK model [28] was conceived to describe EMFFs in the space-like region. To extend this model also in the time-like region, we have revised the definition of the vector meson propagators, we have included energy-dependent widths and then regularized the obtained expressions to eliminate unwanted poles.

Two possible forms of energy-dependent widths have been discussed: case $=1$, the minimal alteration from the non-relativistic $\mathrm{BW}$ formula, and case $=s$ derived from relativistic perturbation theory. The resulting modification in the space-like region, due to the more complex analytic structure of propagators, is minor and affected the fit there very little.

Figures $3-\square$ and table 3 show the results of the global, space-like and time-like, fit to the EMFFs of the nucleons, the outcome is satisfactory, even in light of a so etherogenous set of data.

The $\chi^{2}$ contributions from each space-like EMFF differ little between case $=1$ and case $=s$, see also the normalized contributions, $\chi_{i}^{2} / N_{i}$, reported in figs. $3-\square$, and are approximately the same as 
in the space-like only fit of Ref. [15].

In the time-like region, the quality of the fit to the effective proton and neutron FFs is poorer when the $B A B A R$ data [24] are included than when that set of data is omitted.

Figure $\square$, for the ratio $R_{p}$, indicates how new data at higher momentum-transfers could discriminate among the different cases.

Figure 10, for the neutron effective FF, is also extended in energy for the same reason. It clearly shows that at higher energy case $=1$ no $B A B A R$ could be discriminated from the other three fits by moderately precise data.

Figure $\square$ shows the modulus of the time-like ratio $G_{E}^{p} / G_{M}^{p}$. Even though there are two sets of data on this quantity, one of them [24] is shown in the figure, we decided to not include these data in the fit, hence the curves are predictions. Finally, in fig. 12 , is represented the relative phase between the electric and magnetic proton FFs, $G_{E}^{p}$ and $G_{M}^{p}$, in the time-like region. There are no data on such an observable. So, also in this case, the curves represent predictions of the model. It is interesting to notice that these time-like observables, as a consequence of the complex structure of the EMFFs, are even more sensitive of the space-like ones, to the different cases.

Precise data on these time-like observables will be effective in disentangle and selecting models.

\section{References}

[1] L. L. Foldy, Phys. Rev. 87, 688 (1952).

[2] L. N. Hand, D. G. Miller and R. Wilson, Rev. Mod. Phys. 35, 335 (1963).

[3] C. F. Perdrisat, V. Punjabi and M. Vanderhaeghen, Prog. Part. Nucl. Phys. 59, 694 (2007) [arXiv:hep-ph/0612014].

[4] V. Matveev et al., Nuovo Cimento Lett. 7 (1973) 719.

[5] S. J. Brodsky and G. R. Farrar, Phys. Rev. Lett. 31, 1153 (1973);

G. P. Lepage and S. J. Brodsky, Phys. Rev. D 22, 2157 (1980).

[6] E. C. Titchmarsh, Theory of functions, Oxford University Press, London, 1939.

[7] S. Pacetti, Chin. Phys. C 34, 874 (2010) [arXiv:1012.1232 [hep-ph]].

[8] E. L. Lomon and S. Pacetti, Phys. Rev. D 85, 113004 (2012) [Erratum-ibid. D 86, 039901 (2012)] [arXiv:1201.6126 [hep-ph]].

[9] F. Iachello, A. D. Jackson, and A. Landé, Phys. Lett. B 43, 191 (1973).

[10] M. F. Gari and W. Krümpelmann, Phys. Lett. B 274, 159 (1992); 282, 483 (1992).

[11] E. L. Lomon, arXiv:nucl-th/0609020; Phys. Rev. C 66, 045501 (2002); Prepared for 9th International Conference on the Structure of Baryons (Baryons 2002), Newport News, Virginia (2002); Phys. Rev. C 64, 035204 (2001).

[12] E. Tomasi-Gustafsson, F. Lacroix, C. Duterte and G. I. Gakh, Eur. Phys. J. A 24, 419 (2005) [arXiv:nucl-th/0503001].

[13] R. J. Eden, P. V. Landshoff, D. I. Olive, and J. C. Polkinghorne, The Analytic S-matrix (Cambridge University Press, Cambridge, England, 1966);

G. F. Chew, The Analytic S-Matrix (Benjamin, New York, 1966). 
[14] J. Beringer et al. (Particle Data Group), Phys. Rev. D 86, 010001 (2012)

[15] C. Crawford et al., Phys. Rev. C 82, 045211 (2010) [arXiv:1003.0903 [nucl-th]].

[16] A. Antonelli et al. [FENICE Collaboration], Nucl. Phys. B 517, 3 (1998).

[17] B. Delcourt et al. [DM1 Collaboration], Phys. Lett. B 86, 395 (1979).

[18] D. Bisello et al. [DM2 Collaboration], Nucl. Phys. B 224, 379 (1983).

[19] M. Ablikim et al. [BES Collaboration], Phys. Lett. B 630, 14 (2005) [arXiv:hep-ex/0506059].

[20] T. K. Pedlar et al. [CLEO Collaboration], Phys. Rev. Lett. 95, 261803 (2005) [arXiv:hep-ex/0510005].

[21] G. Bardin et al. [LEAR Collaboration], Nucl. Phys. B 411 (1994) 3.

[22] T. A. Armstrong et al. [E760 Collaboration], Phys. Rev. Lett. 70, 1212 (1993).

[23] M. Ambrogiani et al. [E835 Collaboration], Phys. Rev. D 60, 032002 (1999).

[24] B. Aubert et al. [BABAR Collaboration], Phys. Rev. D 73, 012005 (2006) [arXiv:hep-ex/0512023].

[25] V. V. Bytev, E. A. Kuraev, E. Tomasi-Gustafsson and S. Pacetti, Phys. Rev. D 84, 017301 (2011) [arXiv:1103.4470 [hep-ph]].

[26] R. B. Baldini, S. Pacetti, A. Zallo and A. Zichichi, Eur. Phys. J. A 39, 315 (2009) [arXiv:0711.1725 [hep-ph]]; R. B. Ferroli, S. Pacetti and A. Zallo, Eur. Phys. J. A 48, 33 (2012) [arXiv:1008.0542 [hep-ph]].

[27] J. Haidenbauer, H. W. Hammer, U. G. Meissner and A. Sibirtsev, Phys. Lett. B 643, 29 (2006) [arXiv:hep-ph/0606064].

[28] E. L. Lomon, arXiv:nucl-th/0609020;

E. L. Lomon, Phys. Rev. C 66, 045501 (2002) [arXiv:nucl-th/0203081];

E. L. Lomon, Prepared for 9th International Conference on the Structure of Baryons (Baryons 2002), Newport News, Virginia, 3-8 March 2002;

E. L. Lomon, Phys. Rev. C 64, 035204 (2001) [arXiv:nucl-th/0104039]. 